\title{
Experience with concurrent chemoradiotherapy treatment in advanced cervical cancer: Results from a hospital in Argentina
}

\section{María Eugenia Giavedoni ${ }_{1}$, Lucas Staringer ${ }_{2}$, Rosa Garrido ${ }_{1}$, Cintia Bertoncini ${ }_{2}$, Mabel Sardi ${ }_{2}$, Myriam Perrotta}

1Department of Gynecology, Hospital Italiano of Buenos Aires, Buenos Aires C1199 ABH, Argentina

2Department of Radiation Oncology, Hospital Italiano of Buenos Aires, Buenos Aires C1199 ABH, Argentina

\section{Objetive}

To describe our experience with concurrent chemoradiotherapy using three-dimensional conformal radiotherapy (3D-CRT) and high-dose-rate intracavitary brachytherapy with weekly cisplatin in the treatment of patients with locally advanced cervical cancer.

\section{Methods}

Forty-three patients were identified between January 2009 and December 2015 Their medical records were retrospectively reviewed, and data on patient

characteristics, tumour, treatment and

toxicities were collected and analysed.

\section{Results}

The median age was $\mathbf{4 5}$ years (interquartile range (IQR): 26).

The median tumour size was $45 \mathrm{~mm}$ (IQR: 20).

38 patients ( $88 \%$ ) had a cervical tumour with a size of $\geq 40 \mathrm{~mm}$.

The median cervical tumour size evaluated by magnetic resonance imaging (MRI) was 52 mm (IQR: 17).

22 patients (51\%) had enlarged lymph nodes on MRI ( $\geq 10 \mathrm{~mm}$ ).

MRI demonstrated the involvement of the parametrium in 29 patients (67\%).

15 patients had positive para-aortic nodes (36\%).

The median total treatment time was 58 days (IQR: 20).

16 patients (39\%) received extended-field radiotherapy.

Cisplatin was administered simultaneously for a median of 5 courses.

The median follow-up period was 32 months (IQR: 28 months).

Grade 3 acute toxicity was observed at the gastrointestinal level in 7 patients (16\%).

Late grade $3 / 4$ toxicity was observed in 14 patients (33\%). 7 patients (16\%) persisted with the disease and five died. The local relapse rate was $9 \%$.

11 patients underwent a hysterectomy after treatment.

The disease-free interval was 24.2 months. The 2-year global survival rate was $82.9 \%$.

\section{Conclusion}

Concurrent chemo-radiotherapy appears to be an effective regimen, with acceptable toxicity, for patients with locally advanced cervical cancer.

In spite of being a study with a low number of cases and with a follow-up of only two years, we highlight that the

patients belong to a single institution and have had an exhaustive follow-up with special attention to their therapeutic complications.

We report favourable results regarding radiochemotherapy concurrent with high rate brachytherapy in cervical cancer in locally advanced stages. This concurrent treatment showed good local control, was well tolerated, and had an acceptable percentage of complications.
Baseline characteristics of cervical cancer patients $(n=43)$

\begin{tabular}{|c|c|c|}
\hline \multicolumn{2}{|l|}{ Details } & Measurement \\
\hline \multicolumn{2}{|l|}{ Patients (n) } & 43 \\
\hline \multicolumn{2}{|l|}{ Age (years), median (IQR) $\Delta$} & $45(26)$ \\
\hline \multirow{3}{*}{ Performance status (n/\%) } & 0 & $32 / 74.4$ \\
\hline & 1 & $10 / 23.3$ \\
\hline & 2 & $1 / 2.3$ \\
\hline \multicolumn{2}{|l|}{ Body Mass Index in $\mathrm{kg} / \mathrm{m}^{2}$, median (IQR) $\Delta$} & $23.8(7.5)$ \\
\hline \multirow{2}{*}{ Patient with history of prior surgeries (n/\%) } & No & $30 / 69.8$ \\
\hline & Yes & $13 / 30.2$ \\
\hline \multicolumn{2}{|c|}{ Haemoglobin pretreatment in $\mathrm{g} / \mathrm{dL}$, median (IQR) $\Delta$} & $12(2.1)$ \\
\hline \multicolumn{2}{|l|}{ Creatinine clearance in $\mathrm{mL} / \mathrm{min}$, median (IQR) $\Delta$} & $97(47.7)$ \\
\hline
\end{tabular}

Tumour characteristics of cervical cancer patients $(n=43)$

\begin{tabular}{|c|c|c|}
\hline \multicolumn{2}{|l|}{ Details } & Measurement \\
\hline \multirow{3}{*}{ Histological type (n/\%) } & Squamous & $37 / 86$ \\
\hline & Adenocarcinoma & $4 / 9.3$ \\
\hline & Other & $2 / 4.7$ \\
\hline \multicolumn{2}{|l|}{ Tumour size in $\mathrm{cm}$, median (IQR) $\Delta$} & $45(20)$ \\
\hline \multirow{4}{*}{ Tumour stage (FIGO) (n/\%) } & IB2 & $12 / 27.9$ \\
\hline & IIA2 & $6 / 14.0$ \\
\hline & IIB & $15 / 34.9$ \\
\hline & IIIB & $10 / 23.3$ \\
\hline \multicolumn{2}{|c|}{ Tumour size in images (MRI) in cm, median (IQR) $\Delta$} & $52(17)$ \\
\hline \multirow{2}{*}{$\begin{array}{l}\text { Lymph node involvement in } \\
\text { images (MRI) (n/\%) }\end{array}$} & Negative & $21 / 48.8$ \\
\hline & Positive & $22 / 51.2$ \\
\hline \multirow{2}{*}{$\begin{array}{l}\text { Parametrial infiltration in images } \\
\text { (MRI) (n/\%) }\end{array}$} & Negative & $14 / 32.6$ \\
\hline & Positive & $29 / 67.4$ \\
\hline \multirow{2}{*}{$\begin{array}{l}\text { Pretreatment lumbar aortic } \\
\text { staging (surgery) (n/\%) }\end{array}$} & No & $20 / 46.5$ \\
\hline & Yes & $23 / 53.5$ \\
\hline \multicolumn{2}{|c|}{ Lumbar aortic lymph node involvement (PET/surgery) (n/\%) } & $15 / 36$ \\
\hline
\end{tabular}

Characteristics of concurrent radiochemotherapy in cervical cancer patients $(n=43)$

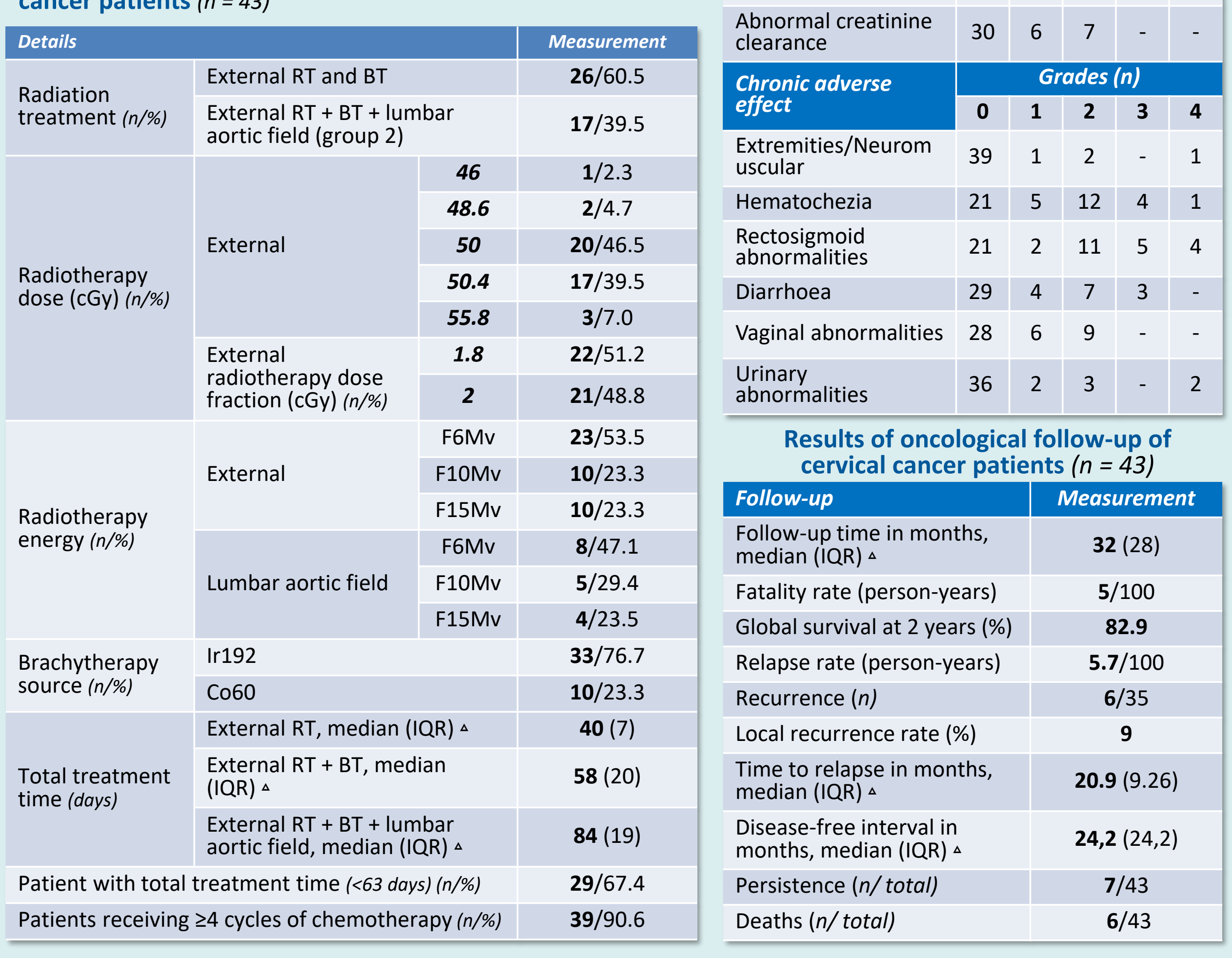

Acute and chronic toxicities profile in cervical cancer patients $(n=43)$

\begin{tabular}{|l|c|c|c|c|c|}
\hline \multirow{2}{*}{ Acute adverse effect } & \multicolumn{5}{|c|}{ Grades (n) } \\
\cline { 2 - 7 } & $\mathbf{0}$ & $\mathbf{1}$ & $\mathbf{2}$ & $\mathbf{3}$ & $\mathbf{4}$ \\
\hline $\begin{array}{l}\text { Lower } \\
\text { gastrointestinal }\end{array}$ & 1 & 5 & 30 & 7 & - \\
\hline Urinary & 1 & 17 & 25 & - & - \\
\hline Asthenia & 1 & 21 & 20 & 1 & - \\
\hline Dermatological & 18 & 23 & 2 & - & - \\
\hline Vomiting & 39 & 3 & 1 & - & - \\
\hline $\begin{array}{l}\text { Anaemia } \\
\text { (haemoglobin) }\end{array}$ & 13 & 19 & 11 & - & - \\
\hline Leukopenia & 14 & 19 & 6 & 4 & - \\
\hline Neutropenia & 33 & 4 & 6 & - & - \\
\hline Thrombocytopenia & 39 & 4 & - & - & - \\
\hline Infectious & 39 & - & 3 & - & 1 \\
\hline Motor neuropathy & 42 & - & - & 1 & - \\
\hline Sensory neuropathy & 39 & - & 2 & 1 & 1 \\
\hline $\begin{array}{l}\text { Abnormal } \\
\text { transaminases }\end{array}$ & 40 & 1 & 2 & - & - \\
\hline Abnormal bilirubin & 43 & - & - & - & - \\
\hline Hyponatremia & 36 & 4 & - & 3 & - \\
\hline $\begin{array}{l}\text { Abnormal creatinine } \\
\text { clearance }\end{array}$ & 30 & 6 & 7 & - & - \\
\hline $\begin{array}{l}\text { Chronic adverse } \\
\text { effect }\end{array}$ & 36 & 2 & 3 & - & 2 \\
\hline $\begin{array}{l}\text { Extremities/Neurom } \\
\text { uscular }\end{array}$ & 39 & 1 & 2 & - & 1 \\
\hline Hematochezia & 21 & 5 & 12 & 4 & 1 \\
\hline $\begin{array}{l}\text { Rectosigmoid } \\
\text { abnormalities }\end{array}$ & 21 & 2 & 11 & 5 & 4 \\
\hline Diarrhoea & 29 & 4 & 7 & 3 & - \\
\hline Vaginal abnormalities & 28 & 6 & 9 & - & - \\
\hline $\begin{array}{l}\text { Urinary } \\
\text { abnormalities }\end{array}$ & $\mathbf{2}$ & $\mathbf{2}$ & $\mathbf{3}$ & $\mathbf{4}$ \\
\hline & & & \\
\hline
\end{tabular}

Results of oncological follow-up of 